\title{
Correction to: Topographic Mapping of Parietal Cortex
}

\section{Summer Sheremata}

\section{Correction to:}

Chapter "Topographic Mapping of Parietal Cortex" in: S. Pollmann, Neuromethods, https://doi.org/10.1007/7657_2019_23

This chapter was inadvertently published with incorrect Tables 1 and 2 . The correct presentation is given here. 


\section{Table 1}

\section{\# Create anatomy file and copy to output directory}

mri_convert $\$\{$ subj_anat_dir $\} /$ brain.mgz $\$\{$ subj_anat_dir $\} / \mathrm{mri} / \mathrm{brain} . n i i$ 3dcopy $\$\{$ subj_anat_dir $\} / \mathrm{mri} /$ brain.nii $\$\{$ subj $\} \_$anat_stripped+orig

\section{\# Motion correct}

touch out.pre_ss_warn.txt

foreach run(\$runs)

3dvolreg -verbose -zpad 1 -base pb00.\$\{subj\}.r01.tcat+orig.'[2]' $\backslash$ -1Dfile dfile.r $\$\{$ run $\} .1 \mathrm{D}$-prefix pb01.\$\{subj\}.r\$\{run\}.volreg $\backslash$ -cubic pb00.\$subj.r\$run.tcat+orig

end

\# Detrend

foreach run(\$runs)

3dTstat -prefix tempMean pb01.\$\{subj\}.run\$ $\{$ run\}.volreg

$3 \mathrm{dDetrend}$-prefix tempDetrend -vector dfile.r\$\{run\}.1D $\backslash$ -polorrt 2 pb01.\$\{subj\}.r\$\{run\}.volreg+orig

3dcalc -a tempDetrend+orig -b tempMean+orig -expr "(a+b)" -float $\backslash$ -prefix pb02.\$\{subj\}.r\$\{run\}.detrended

rm temp*

end

\# Smoothing

foreach run(\$runs)

3dmerge -1blur_fwhm 4.0 -doall -prefix pb03.\$\{subj\}.r\$ $\$$ run $\}.$ smoothing $\backslash$ $\mathrm{pb} 02 . \$\{$ subj $\} . r \$\{$ run $\}$. detrend + orig

end 


\section{Table 1}

(continued)

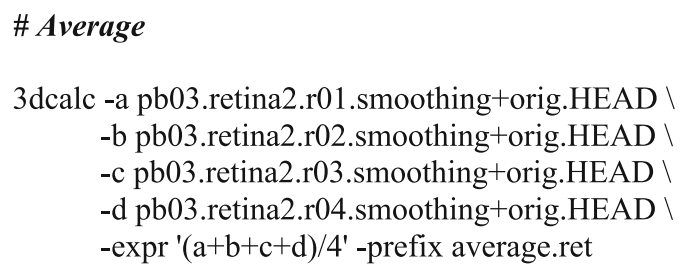

\# Automask and remove mean

3dcopy average.ret AFNI_pRF+orig.BRIK

3dTstat -mean -prefix AFNI_pRFmean AFNI_pRF+orig.BRIK

3dAutomask -prefix automask AFNI_pRF+orig.BRIK

3dcalc -a AFNI_pRF+orig. -b AFNI_pRFmean+orig. -c automask+orig. I -expr $100 * c^{*}(a-b) / b$ ' -prefix e.scale.demean

\section{\# Run model}

3dNLfim -input e.scale.demean+orig -mask automask+orig -noise Zero \ -signal Conv_PRF

-sconstr $0-10.010 .0 \quad$ I

-sconstr $1-1.01 .0$

-sconstr $2-1.01 .0$

-sconstr 30.01 .0

-BOTH -nrand 10000 -nbest 5 -bucket 0 Buckslow.PRF -snfit snfitslow.PRF

3dcalc -a Buckslow.PRF+orig'[1]' -b Buckslow.PRF+orig'[2]' -expr 'sqrt(a^2+b^2)' । -prefix polarslow.m

3dcalc -a Buckslow.PRF+orig.'[1]' -b Buckslow.PRF+orig.'[2]' -expr 'atan2(a,-b)' । -prefix polarslow.ph

3dbucket -glueto Buckslow.PRF+orig. polarslow.m+orig. polarslow.ph+orig. 


\section{Table 2}

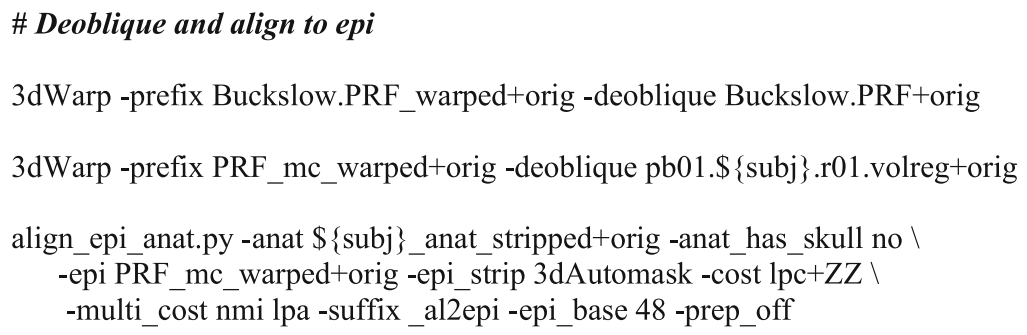

Also, the chapter has been published without updating the following correction:

p. 12, First paragraph last sentence has been amended to 'Here I have inverted the left hemisphere so that the colors in both hemispheres represent the same locations vertically.' 